\title{
Factors Associated with Cigarette Smoking Cessation in Lao People's Democratic Republic: Results from the 2015 National Adult Tobacco Survey
}

\author{
Thanh Cong Bui ${ }^{1, *}$, Phonepadith Xangsayarath ${ }^{2}$, Daovieng Douangvichith ${ }^{3}$, \\ Latsamy Siengsounthone ${ }^{4}$, Khatthanaphone Phandouangsy ${ }^{5}$, Ly Thi-Hai Tran ${ }^{6}$ and \\ Michael S. Businelle 1 \\ 1 Department of Family and Preventive Medicine, Stephenson Cancer Center, \\ Oklahoma Tobacco Research Center, The University of Oklahoma Health Sciences Center, \\ Oklahoma City, OK 73104, USA; Michael-Businelle@ouhsc.edu \\ 2 National Center for Laboratory and Epidemiology, Ministry of Health of Lao PDR, \\ Vientiane, Laos; phonepadithxangsayarath@gmail.com \\ 3 Ministry of Health of Lao PDR, Vientiane, Laos; ddaovieng2@gmail.com \\ 4 Lao Tropical and Public Health Institute, Ministry of Health of Lao PDR, \\ Vientiane, Laos; slatsamy@yahoo.com \\ 5 Secretariat of the National Tobacco Control Committee, Department of Hygiene and Health Promotion, \\ Ministry of Health of Lao PDR, Vientiane, Laos; tphandouangsy@yahoo.com \\ 6 Oklahoma Medical Research Foundation, Oklahoma City, OK 73104, USA; Ly-Tran@omrf.org \\ * Correspondence: thanh-c-bui@ouhsc.edu or thanh.bui@aya.yale.edu
}

Received: 12 May 2020; Accepted: 22 June 2020; Published: 9 July 2020

\begin{abstract}
Cigarette smoking represents a major public health problem in Lao People's Democratic Republic (Lao PDR). This study aims to examine factors associated with cigarette smoking cessation attempts and intention to quit. Data were from the Lao National Adult Tobacco Survey that consisted of 7562 participants $\geq 15$ years old. Multivariable logistic regression models were used to evaluate the associations, adjusted for sex, age groups, education level, income per day, and smoking frequency. Results show that past quit attempts were associated with visiting a healthcare provider in the past year (adjusted odds ratio [AOR]: 1.74, 95\% confidence intervals [CI]: 1.28-2.35), home smoking bans (AOR: 5.52, 95\% CI: 2.13-14.33), noticing media-based messages informing the dangers of smoking or encouraging quitting (AOR: $3.25,95 \%$ CI: 2.28-4.63), noticing health warnings on cigarette packages in the past 30 days (AOR: $3.33,95 \% \mathrm{CI}: 2.21-5.03$ ), and believing that smoking is seriously harmful to their health (AOR: $3.45,95 \%$ CI: 1.24-9.57). The Lao PDR government should continue implementing tobacco control policies that demonstrated associations with cessation attempts or intention to quit, such as smoke-free environments and required health warnings on cigarette packages. Tobacco cessation treatment programs are pressingly needed in Lao PDR.
\end{abstract}

Keywords: tobacco use; cigarette smoking; cessation; Lao People's Democratic Republic

\section{Introduction}

Cigarette smoking represents a major public health problem in developing countries $[1,2]$ and in Lao People's Democratic Republic (Lao PDR) specifically. In our previous report of the 2015 Lao National Adult Tobacco Survey (NATS), the prevalence of cigarette smoking was $48.9 \%$ in men and $5.3 \%$ in women [3]. Current tobacco use was more prevalent among groups with older ages and groups with lower education levels.

When the NATS 2015 data were collected, Lao PDR had several national policies and strategies for tobacco control, following the World Health Organization's MPOWER measures (Monitoring 
tobacco use and tobacco control policies; Protecting people from the dangers of tobacco smoke [i.e., smoke-free policies]; Offering help to quit tobacco [i.e., cessation programs]; Warning the public about the dangers of tobacco, including health warnings on cigarette packages and anti-tobacco mass media campaigns; Enforcing bans on advertising, promotion and sponsorship; and Raising tobacco taxes) [4-6]. Specifically, smoke-free environments were enforced in many public facilities in Lao PDR, including healthcare facilities, educational facilities, and government-owned offices and workplaces $[4,7]$. Tobacco taxes were levied, though total tobacco prices after taxes in Lao PDR were still lower than those in other Southeast Asian countries [4,8,9]. Tobacco advertising was comprehensively banned, and health warning images and messages on cigarette packages were required. The National Tobacco Control Committee (NTCC) of Lao PDR coordinated with several ministries to implement and enforce all of these measures. Despite these achievements, Lao PDR lacked some critical programs for tobacco control. Specifically, there was no large-scale tobacco treatment program such as a national toll-free telephone quitline [4]. Although a few small-scale pilot telephone or in-person counseling programs were tested, funding limitations prevented large-scale or nationwide implementation. Nicotine replacement therapy and other medications to treat tobacco dependence, most of which were unofficially imported, were available over-the-counter at some retail pharmacies; however, they were available only in large cities and were not covered by national health insurance.

Using NATS 2015 data, this study aims to examine individual-level factors that were associated with successful cigarette smoking cessation, making a quit attempt, and intention to quit in the future. Findings of this study will provide useful information to reinforce or modify tobacco control strategies in Lao PDR and other countries in the region.

\section{Materials and Methods}

The Lao NATS 2015 used the Global Adult Tobacco Survey (GATS) standard protocol [10], including sampling strategies, selected age range, and measures. Briefly, the Lao NATS consisted of a nationally representative sample of 7562 participants $\geq 15$ years old recruited nationwide through a stratified 2-stage cluster sampling approach. Provinces served as strata $(n=18)$, and villages or comparable urban administrative units served as primary sampling units (PSU) in each stratum. At the first stage, PSUs were selected by using the probability proportional to size method. At the second stage, 20 households were selected from each PSU through a circular systematic sampling method with a randomly selected starting household (total sample: 2969 households). All eligible people $\geq 15$ years old in selected households were invited to participate (participation rate: $85 \%$, with an average of 2.5 persons/household participated). In Lao PDR, people $\geq 15$ years old can give consent and are expected to complete compulsory education, which lasts 9 years from age 6 to age 14 . The survey included questions about demographic characteristics, various tobacco use practices, smoking characteristics, awareness of harms caused by tobacco, exposure to secondhand smoke, and beliefs related to tobacco use (e.g., smoking causes illnesses or cigarettes' price should be increased). The MPOWER measures were used as a framework for the survey questions as well as for our analyses. The questionnaires were administered by CommCare software on tablets. All interviewers were thoroughly trained to conduct the survey to ensure standardization. Further details of the Lao NATS 2015 can be found in our previous publication [3]. The study received ethical review and approval from the Lao National Ethics Committee for Health Research (IRB00006227).

The main dependent variables in this analysis were (i) being a former cigarette smoker (i.e., already quit), (ii) current smokers who made a quit attempt in the past year (i.e., past quit attempts), and (iii) current smokers who did not make a quit attempt in the past year but planned to quit in the future (i.e., intention to quit). Current cigarette smokers were participants who answered "daily" or "less than daily" to the question, "Do you currently smoke tobacco daily, less than daily, or not at all?" and answered $\geq 1$ to a question about the number of cigarettes smoked per week. Former cigarette smokers were those who did not currently smoke but answered "daily" or "less than daily" to the question, "In the past, have you smoked tobacco on a daily basis, less than daily, or not at all?" and 
answered $\geq 1$ to a question about the number of cigarettes that participants smoked per week in the past. Making a quit attempt in the past year was defined as using any methods to try to stop smoking during the past 12 months, including counseling, pharmacotherapy, traditional medicine, and self-quitting (cold turkey). Based on the question, "Which of the following best describes your current thinking about quitting smoking?" current smokers who responded, "planning to quit within the next month," "thinking about quitting within the next 12 months," or "I will quit someday but not within the next 12 months," were considered planning to quit in the future; while those who responded, "I am not interested in quitting," were considered not planning to quit. Unless specified, responses/values of almost all independent variables or covariates (e.g., except age groups) listed in the tables are in their original forms of measures.

We used Stata 14.2 (StataCorp, College Station, TX, USA) to perform statistical analyses and accounted for the complex sampling design and sampling weights. We used appropriate tests (e.g., chi-square, binary logistic regression) to examine bivariate associations between dependent variables and sociodemographic and behavioral characteristics of interest. We used different multivariable logistic regression models to evaluate associations between each dependent and independent variable, adjusted for sex, age groups, education level, income per day, and smoking frequency. All $p$-values were two-tailed and were considered statistically significant if $<0.05$.

\section{Results}

Table 1 displays the sociodemographic and behavioral characteristics of current and former smokers in Lao PDR. Most current smokers were male (89\%), lived in rural areas (77\%), smoked daily $(92 \%)$, and more than half of smokers $(61 \%)$ had an average income below the poverty line. Most current smokers were married $(87 \%)$ and were allowed to smoke at home (97\%). Sixty three percent of former smokers and $45 \%$ of current smokers agreed that the price of cigarettes should be raised to encourage cessation.

Former smokers accounted for $19 \%$ of all ever smokers (Table 2). Among current smokers, $29 \%$ made a quit attempt in the past year. Common methods or aids used to quit included unaided smoking cessation ( $\sim 90 \%$ for both former and current smokers), counseling ( $5 \%$ for former smokers and $9 \%$ for current smokers), switching to tobacco chewing ( $8 \%$ in former smokers and $1 \%$ in current smokers), and nicotine replacement therapy or other prescribed medications ( $1 \%$ in former smokers and $3 \%$ in current smokers). Among current smokers who did not make a quit attempt in the past year, $11 \%$ planned to quit in the future.

Compared with current smokers, former smokers were more likely to visit a healthcare provider in the past year (adjusted odds ratio [AOR]: 1.86, 95\% confidence interval [CI]: 1.23-2.80), less likely to be exposed to secondhand smoking at indoor workplaces in the past 30 days (AOR: $0.33,95 \% \mathrm{CI}$ : 0.15-0.71), more likely to have a smoking ban in place at home (AOR: 4.86, 95\% CI: 3.42-6.92), and more likely to believe that smoking causes illnesses (AOR: 1.79, 95\% CI: 1.04-3.08). Among current smokers, past quit attempts were associated with visiting a healthcare provider in the past year (AOR: $1.74,95 \%$ CI: 1.28-2.35), more likely to have a smoking ban in place at home (AOR: 5.52, 95\% CI: 2.13-14.33), more likely to report noticing media-based messages informing the dangers of smoking or encouraging quitting (AOR: 3.25, 95\% CI: 2.28-4.63), more likely to report noticing health warnings on cigarette packages in the past 30 days (AOR: 3.33, 95\% CI: 2.21-5.03), and more likely to believe that smoking is seriously harmful to their health (AOR: 3.45, 95\% CI: 1.24-9.57). Among current smokers who did not make a quit attempt in last year, intention to quit in the future was associated with noticing media-based messages informing the dangers of smoking or encouraging quitting (AOR: $2.83,95 \%$ CI: 1.34-5.96), noticing health warnings on cigarette packages (AOR: 8.31, 95\% CI: 2.88-23.96), belief that smoking causes illnesses (AOR: 5.06, 95\% CI: 1.29-14.97), and non-belief that smoking made a real man (AOR: $0.34,95 \%$ CI: 0.12-0.96). There were no significant interactions among examined independent variables. 
Table 1. Demographic characteristics of adult cigarette smokers, Lao People's Democratic Republic, 2015 , weighted \% (95\% CI).

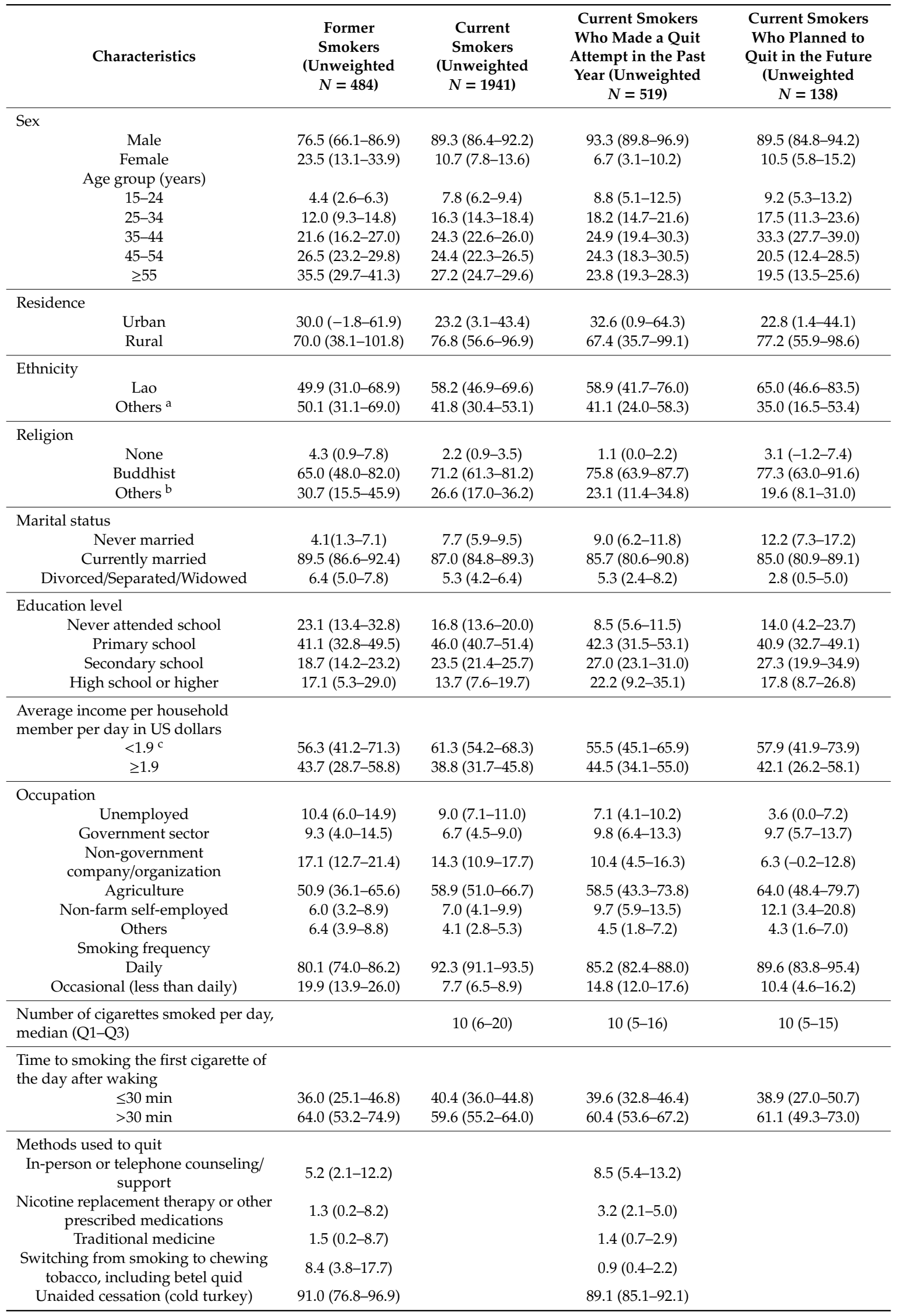


Table 1. Cont.

\begin{tabular}{|c|c|c|c|c|}
\hline Characteristics & $\begin{array}{c}\text { Former } \\
\text { Smokers } \\
\text { (Unweighted } \\
N=484 \text { ) }\end{array}$ & $\begin{array}{c}\text { Current } \\
\text { Smokers } \\
\text { (Unweighted } \\
N=1941 \text { ) }\end{array}$ & $\begin{array}{l}\text { Current Smokers } \\
\text { Who Made a Quit } \\
\text { Attempt in the Past } \\
\text { Year (Unweighted } \\
\quad N=519 \text { ) }\end{array}$ & $\begin{array}{l}\text { Current Smokers } \\
\text { Who Planned to } \\
\text { Quit in the Future } \\
\text { (Unweighted } \\
\quad N=138 \text { ) }\end{array}$ \\
\hline \multicolumn{5}{|l|}{$\begin{array}{l}\text { Visited a health-care provider during } \\
\text { the past } 12 \text { months }\end{array}$} \\
\hline \multicolumn{5}{|l|}{$\begin{array}{l}\text { Ever received advice to quit smoking } \\
\text { from health care providers }\end{array}$} \\
\hline No & $75.9(61.0-90.7)$ & $80.5(76.0-85.1)$ & $75.5(69.9-81.0)$ & $86.4(72.1-100.8)$ \\
\hline Yes & $24.1(9.3-39.0)$ & $19.5(15.0-24.0)$ & $24.5(19.0-30.1)$ & $13.6(-0.8-27.9)$ \\
\hline \multicolumn{5}{|l|}{$\begin{array}{l}\text { Exposed to secondhand smoking at } \\
\text { indoor workplace in the past } 30 \text { days }\end{array}$} \\
\hline \multicolumn{5}{|l|}{$\begin{array}{l}\text { Exposed to secondhand smoking at } \\
\text { indoor public places (including } \\
\text { buildings, healthcare facilities, } \\
\text { restaurants, food store, and public } \\
\text { transportation vehicles) in the past } 30 \\
\text { days }\end{array}$} \\
\hline No & $62.3(55.5-69.2)$ & $64.9(58.3-71.6)$ & $63.0(58.7-67.3)$ & $56.0(44.6-67.5)$ \\
\hline Yes & $37.7(30.8-44.5)$ & $35.1(28.5-41.7)$ & $37.0(32.7-41.3)$ & $44.0(32.5-55.4)$ \\
\hline \multicolumn{5}{|l|}{ Smoking rule at home } \\
\hline Allowed & $86.8(76.4-97.2)$ & $97.4(95.1-99.9)$ & $94.1(89.5-98.6)$ & 100.0 \\
\hline Not allowed & $13.2(2.8-23.6)$ & $2.6(0.2-5.0)$ & $5.9(1.4-10.5)$ & 0 \\
\hline \multicolumn{5}{|l|}{$\begin{array}{l}\text { Noticed media-based messages } \\
\text { informing the dangers of smoking or } \\
\text { encouraging quitting in the past } 30 \\
\text { days }\end{array}$} \\
\hline \multicolumn{5}{|l|}{$\begin{array}{l}\text { Noticed health warnings on cigarette } \\
\text { packages in the past } 30 \text { days }\end{array}$} \\
\hline No & & $29.0(25.8-32.3)$ & $16.4(11.9-20.8)$ & $6.7(2.6-10.9)$ \\
\hline Yes & & $71.0(67.7-74.2)$ & $83.6(79.2-88.1)$ & $93.3(89.1-97.4)$ \\
\hline \multicolumn{5}{|l|}{$\begin{array}{l}\text { Believed that smoking is harmful to } \\
\text { participants' health }\end{array}$} \\
\hline No, not harmful & $6.6(4.2-10.3)$ & $3.5(2.8-4.5)$ & $1.9(0.5-0.5)$ & $2.3(0.5-5.1)$ \\
\hline $\begin{array}{c}\text { Yes, a little harmful or moderately } \\
\text { harmful }\end{array}$ & $20.1(15.4-25.7)$ & $34.0(30.3-38.0)$ & $19.7(12.7-12.7)$ & $23.6(8.0-39.3)$ \\
\hline Yes, seriously harmful & $73.3(65.9-79.5)$ & $62.5(58.5-66.2)$ & $78.4(71.2-71.2)$ & $74.1(58.6-89.5)$ \\
\hline \multicolumn{5}{|l|}{$\begin{array}{l}\text { Believed that smoking causes illnesses } \\
\text { (including bronchitis, lung cancer, } \\
\text { or heart diseases) }\end{array}$} \\
\hline No & $16.3(10.4-24.7)$ & $27.0(20.7-34.4)$ & $22.1(15.4-15.4)$ & $7.4(1.3-16.1)$ \\
\hline Yes & $83.7(75.3-89.6)$ & $73.0(65.7-79.3)$ & $77.9(71.2-71.2)$ & $92.6(83.9-101.3)$ \\
\hline \multicolumn{5}{|l|}{$\begin{array}{l}\text { Believed that it is a sin for a cigarette } \\
\text { or pipe smoker to produce smoke that } \\
\text { is inhaled by other persons. }\end{array}$} \\
\hline No & $12.8(8.8-18.3)$ & $14.0(11.8-16.6)$ & $13.9(11.1-11.1)$ & $7.9(3.5-12.3)$ \\
\hline Yes & $87.2(81.7-91.2)$ & $86.0(83.4-88.2)$ & $86.1(83.3-83.3)$ & $92.1(87.7-96.5)$ \\
\hline \multicolumn{5}{|l|}{$\begin{array}{l}\text { Believed that "A man who does not } \\
\text { smoke is not a real man" }\end{array}$} \\
\hline \multicolumn{5}{|l|}{$\begin{array}{l}\text { Believed that the price of cigarettes } \\
\text { should be raised to encourage people } \\
\text { to stop smoking }\end{array}$} \\
\hline
\end{tabular}

\footnotetext{
${ }^{a}$ Including PhouThai, Khermou, Khamu, Khmu, Leu, Mong, etc. ${ }^{b}$ Including Christian, Pee, Phi, Phy, Pi, etc.

c International poverty line (http://povertydata.worldbank.org/poverty/country/LAO).
} 
Table 2. Factors associated with smoking cessation and cessation intention among adult cigarette smokers, Lao People's Democratic Republic, 2015.

\begin{tabular}{|c|c|c|c|c|c|c|c|c|c|}
\hline \multirow[t]{2}{*}{ Characteristics } & \multicolumn{3}{|c|}{$\begin{array}{l}\text { Former Smokers (Unweighted } N=484 \text { ) vs. Current } \\
\text { Smokers (Unweighted } N=1941) \\
\text { (Total Sample: Ever Smoked Cigarettes, } N=2425 \text { ) }\end{array}$} & \multicolumn{3}{|c|}{$\begin{array}{l}\text { Current Smokers Who Made a Quit Attempt in the Past } \\
\text { Year (Unweighted } N=519 \text { ) vs. Who Did not (Unweighted } \\
\qquad N=1281 \text { ) } \\
\text { (Total Sample: Current Smokers Who Answered the } \\
\text { Question about Past Quit Attempts, } N=1800 \text { ) }\end{array}$} & \multicolumn{3}{|c|}{$\begin{array}{l}\text { Current Smokers Who Planned to Quit in the Future } \\
\text { (Unweighted } N=138 \text { ) vs. Those Who Did not } \\
\text { (Unweighted } N=1141 \text { ) } \\
\text { (Total Sample: Current Smokers Who Did not Make a } \\
\text { Quit Attempt Last Year, } N=1279 \text { ) }\end{array}$} \\
\hline & $\begin{array}{l}\text { Weighted \% of } \\
\text { Former Smoker } \\
\quad(95 \% \mathrm{CI})\end{array}$ & $\begin{array}{l}\text { Unadjusted OR } \\
\quad(95 \% \mathrm{CI})\end{array}$ & $\begin{array}{l}\text { Adjusted OR }{ }^{\mathrm{d}} \\
\quad(95 \% \mathrm{CI})\end{array}$ & $\begin{array}{l}\text { Weighted \% of } \\
\text { Those Who } \\
\text { Made A Quit } \\
\text { Attempt Last } \\
\text { Year }(95 \% \text { CI) }\end{array}$ & $\begin{array}{l}\text { Unadjusted OR } \\
\quad(95 \% \mathrm{CI})\end{array}$ & $\begin{array}{c}\text { Adjusted OR }{ }^{\mathrm{d}}(95 \\
\% \mathrm{CI})\end{array}$ & $\begin{array}{l}\text { Weighted \% of } \\
\text { Those Who } \\
\text { Planned to } \\
\text { Quit }(95 \% \text { CI) }\end{array}$ & $\begin{array}{l}\text { Unadjusted OR } \\
\quad(95 \% \mathrm{CI})\end{array}$ & $\begin{array}{l}\text { Adjusted OR }{ }^{d} \\
\quad(95 \% \text { CI })\end{array}$ \\
\hline Total & $19.2(15.4-23.8)$ & & & $28.6(23.7-34.0)$ & & & $10.6(7.9-13.9)$ & & \\
\hline \multicolumn{10}{|l|}{ Sex } \\
\hline Male & $17.0(12.9-21.9)$ & 1 & 1 & $29.9(24.6-35.8)$ & 1 & 1 & $10.8(8.1-14.3)$ & 1 & 1 \\
\hline Female & $34.4(24.8-45.3)$ & $2.56(1.59-4.14)^{* * *}$ & $1.97(1.04-3.72) *$ & $17.6(12.3-24.7)$ & $0.50(0.30-0.86)^{*}$ & $0.86(0.40-1.83)$ & $8.9(5.9-13.4)$ & $0.81(0.53-1.25)$ & $1.53(0.80-2.95)$ \\
\hline \multicolumn{10}{|l|}{ Age group (years) } \\
\hline $15-24$ & $11.9(7.3-19.0)$ & 1 & 1 & $32.1(25.8-39.1)$ & 1 & 1 & $13.0(8.5-19.3)$ & 1 & 1 \\
\hline $25-34$ & $14.9(10.0-21.7)$ & $1.29(0.82-2.05)$ & $1.40(0.60-3.31)$ & $32.9(27.0-39.3)$ & $1.04(0.72-1.49)$ & $0.42(0.19-0.91) *$ & $12.5(8.7-17.4)$ & $0.95(0.53-1.71)$ & $0.95(0.40-2.25)$ \\
\hline $35-44$ & $17.5(13.6-22.1)$ & $1.56(0.91-2.68)$ & $1.72(0.55-5.40)$ & $29.2(24.3-34.6)$ & $0.87(0.58-1.30)$ & $0.60(0.25-1.45)$ & $14.6(10.1-20.5)$ & $1.14(0.70-1.86)$ & $1.36(0.56-3.34)$ \\
\hline $45-54$ & $20.5(16.7-25.1)$ & $1.91(1.16-3.13)^{*}$ & $1.92(0.76-4.84)$ & $28.5(19.7-39.3)$ & $0.84(0.47-1.51)$ & $0.56(0.25-1.28)$ & $8.9(5.3-14.6)$ & $0.65(0.33-1.28)$ & $0.85(0.35-2.05)$ \\
\hline$\geq 55$ & $23.7(18.2-30.3)$ & $2.30(1.33-3.96)^{* *}$ & $2.75(1.33-5.70) *$ & $24.5(18.1-32.3)$ & $0.69(0.40-1.18)$ & $0.47(0.20-1.09)$ & $7.0(5.1-9.7)$ & $0.51(0.36-0.71)^{* * *}$ & $0.72(0.27-1.93)$ \\
\hline$P$ trend & & $<0.001$ & $<0.001$ & & 0.113 & 0.421 & & $<0.001$ & 0.161 \\
\hline \multicolumn{10}{|l|}{ Residence } \\
\hline Urban & $23.6(14.7-35.6)$ & 1 & 1 & $41.3(23.1-62.2)$ & 1 & 1 & $13.0(8.0-20.4)$ & 1 & 1 \\
\hline Rural & $17.8(14.6-21.6)$ & $0.70(0.37-1.35)$ & $0.77(0.39-1.52)$ & $24.9(21.6-28.5)$ & $0.47(0.18-1.26)$ & $0.50(0.19-1.37)$ & $10.0(7.1-13.9)$ & $0.74(0.39-1.43)$ & $0.91(0.37-2.20)$ \\
\hline \multicolumn{10}{|l|}{ Ethnicity } \\
\hline Lao & $17.0(11.6-24.2)$ & 1 & & $28.9(21.1-38.2)$ & 1 & & $11.8(8.0-17.3)$ & 1 & \\
\hline Others $^{\text {a }}$ & $22.2(17.3-28.1)$ & $1.40(0.83-2.36)$ & & $28.1(23.5-33.1)$ & $0.96(0.58-1.60)$ & & $8.8(6.3-12.1)$ & $0.72(0.39-1.30)$ & \\
\hline \multicolumn{10}{|l|}{ Religion } \\
\hline None & $32.0(16.1-53.6)$ & 1 & & $13.6(7.7-23.0)$ & 1 & & $12.0(3.3-35.6)$ & 1 & \\
\hline Buddhist & $17.9(13.1-23.8)$ & $0.46(0.18-1.20)$ & & $30.4(24.0-37.8)$ & $2.77(1.29-5.94) *$ & & $11.8(8.4-16.3)$ & $0.98(0.22-4.34)$ & \\
\hline Others ${ }^{b}$ & $21.6(15.7-28.9)$ & $0.59(0.27-1.26)$ & & $24.8(20.0-30.4)$ & $2.10(0.99-4.45)$ & & $7.4(5.4-10.1)$ & $0.59(0.17-2.04)$ & \\
\hline \multicolumn{10}{|l|}{ Marital status } \\
\hline Never married & $11.4(5.4-22.6)$ & 1 & & $34.5(26.1-43.9)$ & 1 & & $18.9(12.5-27.5)$ & 1 & \\
\hline Currently married & $19.7(15.9-24.1)$ & $1.91(1.00-3.65)$ & & $28.1(23.2-33.5)$ & $0.74(0.50-1.10)$ & & $10.2(7.5-13.8)$ & $0.49(0.31-0.79) * *$ & \\
\hline $\begin{array}{c}\text { Divorced/Separated/ } \\
\text { Widowed }\end{array}$ & $22.5(15.4-31.6)$ & $2.25(1.08-4.68)^{*}$ & & $27.4(16.7-41.5)$ & $0.72(0.41-1.25)$ & & $5.2(2.2-11.5)$ & $0.24(0.07-0.81)^{*}$ & \\
\hline \multicolumn{10}{|l|}{ Education level } \\
\hline Never attended school & $24.7(17.9-33.1)$ & 1 & 1 & $14.5(10.9-19.0)$ & 1 & 1 & $7.3(3.8-13.6)$ & 1 & 1 \\
\hline Primary school & $17.5(14.0-21.8)$ & $0.65(0.43-0.99)$ * & $0.93(0.58-1.50)$ & $26.1(23.1-29.2)$ & $2.09(1.51-2.88)^{* * *}$ & $2.40(1.44-4.01)^{* *}$ & $9.0(7.0-11.6)$ & $1.26(0.63-2.50)$ & $1.28(0.55-2.94)$ \\
\hline Secondary school & $15.9(11.5-21.6)$ & $0.58(0.34-0.98)^{*}$ & $1.05(0.60-1.83)$ & $32.7(26.5-39.6)$ & $2.88(1.88-4.41)^{* * *}$ & $2.53(1.38-4.64)^{* *}$ & $13.0(8.5-19.2)$ & $1.90(0.84-4.27)$ & $2.80(1.37-5.72)^{* *}$ \\
\hline High school or higher & $23.0(15.7-32.4)$ & $0.91(0.50-1.67)$ & $1.26(0.75-2.12)$ & $47.9(33.2-62.9)$ & $5.44(2.96-9.99)^{* * *}$ & $4.82(2.14-10.87)^{* *}$ & $19.6(13.0-28.3)$ & $3.09(1.27-7.52) *$ & $3.18(1.21-8.31)$ * \\
\hline$P$ trend & & 0.702 & 0.272 & & $<0.001$ & 0.008 & & 0.008 & $<0.001$ \\
\hline
\end{tabular}


Table 2. Cont.

\begin{tabular}{|c|c|c|c|c|c|c|c|c|c|}
\hline \multirow[t]{2}{*}{ Characteristics } & \multicolumn{3}{|c|}{$\begin{array}{c}\text { Former Smokers (Unweighted } N=484 \text { ) vs. Current } \\
\text { Smokers (Unweighted } N=1941) \\
\text { (Total Sample: Ever Smoked Cigarettes, } N=2425 \text { ) }\end{array}$} & \multicolumn{3}{|c|}{$\begin{array}{l}\text { Current Smokers Who Made a Quit Attempt in the Past } \\
\text { Year (Unweighted } N=519 \text { ) vs. Who Did not (Unweighted } \\
N=1281 \text { ) } \\
\text { (Total Sample: Current Smokers Who Answered the } \\
\text { Question about Past Quit Attempts, } N=1800 \text { ) }\end{array}$} & \multicolumn{3}{|c|}{$\begin{array}{l}\text { Current Smokers Who Planned to Quit in the Future } \\
\text { (Unweighted } N=138 \text { ) vs. Those Who Did not } \\
\text { (Unweighted } N=1141 \text { ) } \\
\text { (Total Sample: Current Smokers Who Did not Make a } \\
\text { Quit Attempt Last Year, } N=1279 \text { ) }\end{array}$} \\
\hline & $\begin{array}{l}\text { Weighted \% of } \\
\text { Former Smoker } \\
\quad(95 \% \mathrm{CI})\end{array}$ & $\begin{array}{l}\text { Unadjusted OR } \\
(95 \% \mathrm{CI})\end{array}$ & $\begin{array}{l}\text { Adjusted OR }{ }^{\mathrm{d}} \\
\quad(95 \% \mathrm{CI})\end{array}$ & $\begin{array}{l}\text { Weighted \% of } \\
\text { Those Who } \\
\text { Made A Quit } \\
\text { Attempt Last } \\
\text { Year }(95 \% \text { CI })\end{array}$ & $\begin{array}{l}\text { Unadjusted OR } \\
\text { (95\% CI) }\end{array}$ & $\begin{array}{c}\text { Adjusted OR }^{\mathrm{d}}(95 \\
\% \mathrm{CI})\end{array}$ & $\begin{array}{l}\text { Weighted \% of } \\
\text { Those Who } \\
\text { Planned to } \\
\text { Quit }(95 \% \mathrm{CI})\end{array}$ & $\begin{array}{l}\text { Unadjusted OR } \\
\text { (95\% CI) }\end{array}$ & $\begin{array}{l}\text { Adjusted OR }{ }^{\mathrm{d}} \\
\quad(95 \% \mathrm{CI})\end{array}$ \\
\hline \multicolumn{10}{|l|}{$\begin{array}{l}\text { Average income per } \\
\text { household member per } \\
\text { day in US dollars }\end{array}$} \\
\hline$<1.9^{\mathrm{c}}$ & $17.6(14.2-21.7)$ & 1 & 1 & $25.2(21.7-28.9)$ & 1 & 1 & $10.1(7.5-13.5)$ & 1 & 1 \\
\hline$\geq 1.9$ & $20.8(15.1-28.0)$ & $1.23(0.84-1.79)$ & $1.17(0.85-1.60)$ & $32.8(23.6-43.5)$ & $1.45(1.03-2.04)$ & $1.21(0.98-1.49)$ & $13.3(7.1-23.4)$ & $1.36(0.67-2.78)$ & $1.26(0.64-2.50)$ \\
\hline \multicolumn{10}{|l|}{ Occupation } \\
\hline Unemployed & $21.6(13.1-33.5)$ & 1 & & $21.9(13.8-32.9)$ & 1 & & $3.8(1.5-9.1)$ & 1 & \\
\hline Government sector & $24.7(14.8-38.2)$ & $1.19(0.83-1.71)$ & & $42.7(33.5-52.3)$ & $2.66(1.64-4.32)^{* * * *}$ & & $19.7(13.1-28.5)$ & $6.27(2.28-17.25)^{* *}$ & \\
\hline $\begin{array}{c}\text { Non-government } \\
\text { company/organization }\end{array}$ & $22.2(16.6-29.0)$ & $1.03(0.65-1.66)$ & & $21.1(10.4-38.1)$ & $0.96(0.61-1.50)$ & & $4.3(1.3-12.9)$ & $1.15(0.35-3.72)$ & \\
\hline Agriculture & $17.1(14.0-20.7)$ & $0.75(0.44-1.29)$ & & $28.3(25.4-31.4)$ & $1.41(0.80-2.50)$ & & $11.4(8.1-15.9)$ & $3.30(1.11-9.81)$ * & \\
\hline $\begin{array}{l}\text { Non-farm } \\
\text { self-employed }\end{array}$ & $17.1(11.6-24.5)$ & $0.75(0.40-1.42)$ & & $38.6(27.1-51.6)$ & $2.25(1.35-3.75) * *$ & & $20.7(10.9-35.7)$ & $6.67(2.17-20.49)^{* *}$ & \\
\hline Others & $27.2(19.6-36.4)$ & $1.36(0.79-2.34)$ & & $32.5(18.2-51.1)$ & $1.72(1.05-2.84)^{*}$ & & $12.2(5.7-24.3)$ & $3.56(1.01-12.54)$ * & \\
\hline \multicolumn{10}{|l|}{ Smoking frequency } \\
\hline Daily & $19.3(15.4-23.8)$ & 1 & 1 & $26.1(21.1-31.8)$ & 1 & 1 & $9.8(7.3-13.0)$ & 1 & 1 \\
\hline $\begin{array}{l}\text { Occasional (less than } \\
\text { daily) }\end{array}$ & $17.1(13.0-22.3)$ & $2.98(1.79-4.96)^{* * *}$ & $3.08(1.73-5.48)$ * & $61.5(50.7-71.3)$ & $4.52(2.66-7.68)^{* * *}$ & $3.77(2.19-6.50)$ * & $31.2(18.9-46.8)$ & $4.17(2.11-8.23)^{* * *}$ & $5.51(2.77-10.93)$ * \\
\hline $\begin{array}{l}\text { Number of cigarettes } \\
\text { smoked per day, } \\
\text { median (Q1-Q3) }\end{array}$ & & & & $10(5-16)$ & $0.97(0.94-0.99)^{* *}$ & & $10(5-15)$ & $0.95(0.93-0.97)^{* * *}$ & \\
\hline \multicolumn{10}{|l|}{$\begin{array}{l}\text { Time to smoking the } \\
\text { first cigarette of the day } \\
\text { after waking }\end{array}$} \\
\hline$\leq 30 \mathrm{~min}$ & $6.3(4.2-9.5)$ & 1 & & $25.4(18.3-34.2)$ & 1 & & $9.3(5.9-14.3)$ & 1 & \\
\hline$>30 \mathrm{~min}$ & $7.5(4.4-12.7)$ & $1.21(0.75-1.94)$ & & $26.7(22.1-31.8)$ & $1.07(0.75-1.52)$ & & $10.2(7.9-13.0)$ & $1.11(0.73-1.68)$ & \\
\hline \multicolumn{10}{|l|}{$\begin{array}{l}\text { Visited a health-care } \\
\text { provider during the } \\
\text { past } 12 \text { months }\end{array}$} \\
\hline No & $15.9(13.0-19.3)$ & 1 & 1 & $25.9(20.7-31.9)$ & 1 & 1 & $10.6(7.8-14.3)$ & 1 & 1 \\
\hline Yes & $30.7(22.7-40.0)$ & $2.34(1.70-3.22)^{* * *}$ & $1.86(1.23-2.80)$ ** & $39.6(33.1-46.5)$ & $1.87(1.37-2.56)^{* * *}$ & $1.74(1.28-2.35)^{* * *}$ & $10.3(6.6-15.8)$ & $0.97(0.58-1.64)$ & $1.07(0.63-1.81)$ \\
\hline
\end{tabular}


Table 2. Cont

\begin{tabular}{|c|c|c|c|c|c|c|c|c|c|}
\hline \multirow[t]{2}{*}{ Characteristics } & \multicolumn{3}{|c|}{$\begin{array}{c}\text { Former Smokers (Unweighted } N=484 \text { ) vs. Current } \\
\text { Smokers (Unweighted } N=1941 \text { ) } \\
\text { (Total Sample: Ever Smoked Cigarettes, } N=2425 \text { ) }\end{array}$} & \multicolumn{3}{|c|}{$\begin{array}{l}\text { Current Smokers Who Made a Quit Attempt in the Past } \\
\text { Year (Unweighted } N=519 \text { ) vs. Who Did not (Unweighted } \\
\qquad=1281 \text { ) } \\
\text { (Total Sample: Current Smokers Who Answered the } \\
\text { Question about Past Quit Attempts, } N=1800 \text { ) }\end{array}$} & \multicolumn{3}{|c|}{$\begin{array}{c}\text { Current Smokers Who Planned to Quit in the Future } \\
\text { (Unweighted } N=138 \text { ) vs. Those Who Did not } \\
\text { (Unweighted } N=1141 \text { ) } \\
\text { (Total Sample: Current Smokers Who Did not Make a } \\
\text { Quit Attempt Last Year, } N=1279 \text { ) }\end{array}$} \\
\hline & $\begin{array}{l}\text { Weighted \% of } \\
\text { Former Smoker } \\
\quad(95 \% \mathrm{CI})\end{array}$ & $\begin{array}{l}\text { Unadjusted OR } \\
\quad(95 \% \text { CI })\end{array}$ & $\begin{array}{l}\text { Adjusted OR }{ }^{\mathrm{d}} \\
\quad(95 \% \mathrm{CI})\end{array}$ & $\begin{array}{l}\text { Weighted \% of } \\
\text { Those Who } \\
\text { Made A Quit } \\
\text { Attempt Last } \\
\text { Year }(95 \% \text { CI })\end{array}$ & $\begin{array}{l}\text { Unadjusted OR } \\
(95 \% \mathrm{CI})\end{array}$ & $\begin{array}{c}\text { Adjusted OR }{ }^{\mathrm{d}}(95 \\
\% \mathrm{CI})\end{array}$ & $\begin{array}{l}\text { Weighted \% of } \\
\text { Those Who } \\
\text { Planned to } \\
\text { Quit }(95 \% \text { CI })\end{array}$ & $\begin{array}{l}\text { Unadjusted OR } \\
\quad(95 \% \mathrm{CI})\end{array}$ & $\begin{array}{l}\text { Adjusted OR }{ }^{\mathrm{d}} \\
\quad(95 \% \mathrm{CI})\end{array}$ \\
\hline \multicolumn{10}{|c|}{$\begin{array}{l}\text { Ever received advice to } \\
\text { quit smoking from } \\
\text { health care providers }\end{array}$} \\
\hline No & $7.6(4.1-13.9)$ & 1 & 1 & $36.9(30.7-43.5)$ & 1 & 1 & $10.6(6.5-16.6)$ & 1 & 1 \\
\hline Yes & $9.8(2.7-29.8)$ & $1.32(0.53-3.29)$ & $0.80(0.41-1.55)$ & $51.3(39.4-63.0)$ & $1.81(1.22-2.67)^{* *}$ & $1.67(0.90-3.12)$ & $9.1(2.6-27.2)$ & $0.85(0.21-3.34)$ & $0.60(0.12-2.92)$ \\
\hline \multicolumn{10}{|c|}{$\begin{array}{l}\text { Exposed to secondhand } \\
\text { smoking at indoor } \\
\text { workplace in the past } \\
30 \text { days }\end{array}$} \\
\hline No & $31.2(20.1-44.9)$ & 1 & 1 & $47.3(28.5-66.8)$ & 1 & 1 & $35.5(23.8-49.2)$ & 1 & 1 \\
\hline Yes & $15.7(10.7-22.5)$ & $0.41(0.20-0.84)^{*}$ & $0.33(0.15-0.71) * *$ & $33.3(26.1-41.3)$ & $0.56(0.20-1.54)$ & $0.45(0.07-2.95)$ & $7.1(3.9-12.7)$ & $0.14(0.05-0.40)^{* *}$ & $0.33(0.06-1.69)$ \\
\hline \multicolumn{10}{|c|}{$\begin{array}{l}\text { Exposed to secondhand } \\
\text { smoking at indoor } \\
\text { public places (including } \\
\text { buildings, healthcare } \\
\text { facilities, restaurants, } \\
\text { food store, and public } \\
\text { transportation vehicles) } \\
\text { in the past } 30 \text { days }\end{array}$} \\
\hline No & $18.6(14.2-24.0)$ & 1 & 1 & $27.9(23.2-33.2)$ & 1 & 1 & $9.1(6.2-13.2)$ & 1 & 1 \\
\hline Yes & $20.4(16.4-25.1)$ & $1.12(0.85-1.48)$ & $1.35(0.93-1.95)$ & $29.7(23.5-36.7)$ & $1.09(0.87-1.37)$ & $0.90(0.69-1.17)$ & $13.3(9.7-17.9)$ & $1.53(0.95-2.45)$ & $1.34(0.79-2.27)$ \\
\hline \multicolumn{10}{|l|}{ Smoking rule at home } \\
\hline Allowed & $17.5(14.5-21.0)$ & 1 & 1 & $27.5(23.3-32.1)$ & 1 & 1 & $10.6(8.0-14.0)$ & 1 & 1 \\
\hline Not allowed & $55.2(49.2-61.1)$ & $5.80(4.31-7.80)^{* * *}$ & $4.86(3.42-6.92) * * *$ & $78.5(68.6-85.9)$ & $9.62(5.22-17.73)^{* * * *}$ & $5.52(2.13-14.33)^{* * *}$ & 0 & & \\
\hline \multicolumn{10}{|c|}{$\begin{array}{l}\text { Noticed media-based } \\
\text { messages informing the } \\
\text { dangers of smoking or } \\
\text { encouraging quitting in } \\
\text { the past } 30 \text { days }\end{array}$} \\
\hline No & $18.2(14.5-22.6)$ & 1 & 1 & $13.5(9.3-19.4)$ & 1 & 1 & $5.7(3.1-10.3)$ & 1 & 1 \\
\hline Yes & $19.8(15.2-25.4)$ & $1.11(0.82-1.50)$ & $1.00(0.72-1.40)$ & $36.8(31.6-42.3)$ & $3.72(2.81-4.92)^{* * *}$ & $3.25(2.28-4.63)^{* * *}$ & $14.2(10.6-18.8)$ & $2.75(1.41-5.38)^{* *}$ & $2.83(1.34-5.96) * *$ \\
\hline
\end{tabular}


Table 2. Cont.

\begin{tabular}{|c|c|c|c|c|c|c|c|c|c|}
\hline \multirow[t]{2}{*}{ Characteristics } & \multicolumn{3}{|c|}{$\begin{array}{c}\text { Former Smokers (Unweighted } N=484 \text { ) vs. Current } \\
\text { Smokers (Unweighted } N=1941 \text { ) } \\
\text { (Total Sample: Ever Smoked Cigarettes, } N=2425 \text { ) }\end{array}$} & \multicolumn{3}{|c|}{$\begin{array}{l}\text { Current Smokers Who Made a Quit Attempt in the Past } \\
\text { Year (Unweighted } N=519 \text { ) vs. Who Did not (Unweighted } \\
N=1281 \text { ) } \\
\text { (Total Sample: Current Smokers Who Answered the } \\
\text { Question about Past Quit Attempts, } N=1800 \text { ) }\end{array}$} & \multicolumn{3}{|c|}{$\begin{array}{c}\text { Current Smokers Who Planned to Quit in the Future } \\
\text { (Unweighted } N=138 \text { ) vs. Those Who Did not } \\
\text { (Unweighted } N=1141 \text { ) } \\
\text { (Total Sample: Current Smokers Who Did not Make a } \\
\text { Quit Attempt Last Year, } N=1279 \text { ) }\end{array}$} \\
\hline & $\begin{array}{l}\text { Weighted \% of } \\
\text { Former Smoker } \\
\quad(95 \% \mathrm{CI})\end{array}$ & $\begin{array}{l}\text { Unadjusted OR } \\
\quad(95 \% \mathrm{CI})\end{array}$ & $\begin{array}{l}\text { Adjusted OR }{ }^{d} \\
\quad(95 \% \mathrm{CI})\end{array}$ & $\begin{array}{l}\text { Weighted \% of } \\
\text { Those Who } \\
\text { Made A Quit } \\
\text { Attempt Last } \\
\text { Year }(95 \% \text { CI) }\end{array}$ & $\begin{array}{l}\text { Unadjusted OR } \\
\quad(95 \% \mathrm{CI})\end{array}$ & $\begin{array}{c}\text { Adjusted OR }{ }^{\mathrm{d}}(95 \\
\% \mathrm{CI})\end{array}$ & $\begin{array}{l}\text { Weighted \% of } \\
\text { Those Who } \\
\text { Planned to } \\
\text { Quit }(95 \% \text { CI) }\end{array}$ & $\begin{array}{l}\text { Unadjusted OR } \\
\text { (95\% CI) }\end{array}$ & $\begin{array}{l}\text { Adjusted OR }{ }^{d} \\
\quad(95 \% \text { CI })\end{array}$ \\
\hline \multicolumn{10}{|l|}{$\begin{array}{l}\text { Noticed health } \\
\text { warnings on cigarette } \\
\text { packages in the past } 30 \\
\text { days }\end{array}$} \\
\hline No & & & & $16.3(12.0-21.7)$ & 1 & 1 & $2.0(1.0-3.8)$ & 1 & 1 \\
\hline Yes & & & & $33.9(28.0-40.3)$ & $2.64(1.81-3.84)^{* * *}$ & $3.33(2.21-5.03)^{* * *}$ & $14.3(10.6-19.0)$ & $8.18(3.99-16.79)^{* * *}$ & $\begin{array}{c}8.31(2.88-23.96) \\
* * *\end{array}$ \\
\hline \multicolumn{10}{|l|}{$\begin{array}{l}\text { Believed that smoking } \\
\text { is harmful to } \\
\text { participants' health }\end{array}$} \\
\hline No, not harmful & $30.8(22.0-41.2)$ & 1 & 1 & $14.8(7.2-28.0)$ & 1 & 1 & $5.7(1.9-15.7)$ & 1 & 1 \\
\hline $\begin{array}{l}\text { Yes, a little harmful or } \\
\text { moderately harmful }\end{array}$ & $12.2(9.5-15.6)$ & $0.31(0.22-0.45)^{* * *}$ & $1.25(0.66-2.36)$ & $16.6(9.3-27.7)$ & $1.14(0.50-2.61)$ & $1.48(0.42-5.29)$ & $6.4(3.1-12.6)$ & $1.13(0.27-4.76)$ & $0.61(0.11-3.50)$ \\
\hline $\begin{array}{c}\text { Yes, seriously harmful } \\
P \text { trend }\end{array}$ & $21.6(17.2-26.9)$ & $\begin{array}{c}0.62(0.37-1.04) \\
0.114\end{array}$ & $\begin{array}{c}1.82(0.84-3.95) \\
0.001\end{array}$ & $36.7(32.7-41.0)$ & $\begin{array}{c}3.34(1.50-7.45)^{* *} \\
0.001\end{array}$ & $\begin{array}{c}3.45(1.24-9.57) * \\
0.001\end{array}$ & $14.6(10.3-20.3)$ & $\begin{array}{c}2.86(0.84-9.68) \\
0.027\end{array}$ & $\begin{array}{c}3.22(0.64-16.19) \\
0.003\end{array}$ \\
\hline \multicolumn{10}{|l|}{$\begin{array}{l}\text { Believed that smoking } \\
\text { causes illnesses } \\
\text { (including bronchitis, } \\
\text { lung cancer, or heart } \\
\text { diseases) }\end{array}$} \\
\hline No & $12.9(8.9-18.4)$ & 1 & 1 & $23.1(16.8-30.7)$ & 1 & 1 & $2.2(0.7-6.5)$ & 1 & 1 \\
\hline Yes & $21.9(17.7-26.8)$ & $1.89(1.29-2.77) * *$ & $1.79(1.04-3.08) *$ & $29.1(22.5-36.7)$ & $1.37(0.87-2.14)$ & $1.30(0.72-2.35)$ & $10.7(6.6-16.7)$ & $5.36(1.38-10.78) *$ & $5.06(1.29-14.97) *$ \\
\hline \multicolumn{10}{|l|}{$\begin{array}{l}\text { Believed that it is a sin } \\
\text { for a cigarette or pipe } \\
\text { smoker to produce } \\
\text { smoke that is inhaled } \\
\text { by other persons }\end{array}$} \\
\hline No & $16.1(10.0-24.9)$ & 1 & 1 & $30.6(23.6-38.6)$ & 1 & 1 & $6.9(4.1-11.6)$ & 1 & 1 \\
\hline Yes & $17.5(13.8-22.1)$ & $1.11(0.75-1.65)$ & $1.33(0.76-2.33)$ & $30.6(25.5-36.2)$ & $1.00(0.82-1.23)$ & $1.00(0.72-1.38)$ & $13.0(9.8-17.1)$ & $2.01(1.06-3.82) *$ & $2.04(0.82-5.09)$ \\
\hline
\end{tabular}


Table 2. Cont.

\begin{tabular}{|c|c|c|c|c|c|c|c|c|c|}
\hline \multirow[t]{2}{*}{ Characteristics } & \multicolumn{3}{|c|}{$\begin{array}{c}\text { Former Smokers (Unweighted } N=484 \text { ) vs. Current } \\
\text { Smokers (Unweighted } N=1941 \text { ) } \\
\text { (Total Sample: Ever Smoked Cigarettes, } N=2425 \text { ) }\end{array}$} & \multicolumn{3}{|c|}{$\begin{array}{l}\text { Current Smokers Who Made a Quit Attempt in the Past } \\
\text { Year (Unweighted } N=519 \text { ) vs. Who Did not (Unweighted } \\
N=1281 \text { ) } \\
\text { (Total Sample: Current Smokers Who Answered the } \\
\text { Question about Past Quit Attempts, } N=1800 \text { ) }\end{array}$} & \multicolumn{3}{|c|}{$\begin{array}{c}\text { Current Smokers Who Planned to Quit in the Future } \\
\text { (Unweighted } N=138 \text { ) vs. Those Who Did not } \\
\text { (Unweighted } N=1141 \text { ) } \\
\text { (Total Sample: Current Smokers Who Did not Make a } \\
\text { Quit Attempt Last Year, } N=1279 \text { ) }\end{array}$} \\
\hline & $\begin{array}{l}\text { Weighted \% of } \\
\text { Former Smoker } \\
\quad(95 \% \mathrm{CI})\end{array}$ & $\begin{array}{l}\text { Unadjusted OR } \\
(95 \% \mathrm{CI})\end{array}$ & $\begin{array}{l}\text { Adjusted OR }{ }^{\mathrm{d}} \\
\quad(95 \% \mathrm{CI})\end{array}$ & $\begin{array}{l}\text { Weighted \% of } \\
\text { Those Who } \\
\text { Made A Quit } \\
\text { Attempt Last } \\
\text { Year }(95 \% \text { CI) }\end{array}$ & $\begin{array}{l}\text { Unadjusted OR } \\
\quad(95 \% \mathrm{CI})\end{array}$ & $\begin{array}{c}\text { Adjusted OR }{ }^{\mathrm{d}}(95 \\
\% \mathrm{CI})\end{array}$ & $\begin{array}{l}\text { Weighted \% of } \\
\text { Those Who } \\
\text { Planned to } \\
\text { Quit }(95 \% \mathrm{CI})\end{array}$ & $\begin{array}{l}\text { Unadjusted OR } \\
\quad(95 \% \mathrm{CI})\end{array}$ & $\begin{array}{l}\text { Adjusted OR }{ }^{d} \\
\quad(95 \% \text { CI })\end{array}$ \\
\hline \multicolumn{10}{|l|}{$\begin{array}{l}\text { Believed that "A man } \\
\text { who does not smoke is } \\
\text { not a real man" }\end{array}$} \\
\hline No & $19.2(14.9-24.2)$ & 1 & 1 & $30.3(25.6-35.5)$ & 1 & 1 & $13.4(9.7-18.1)$ & 1 & 1 \\
\hline Yes & $14.0(10.4-18.6)$ & $0.69(0.54-0.87)^{* *}$ & $0.63(0.47-0.85)$ ** & $30.6(21.8-41.0)$ & $1.01(0.72-1.43)$ & $0.84(0.51-1.38)$ & $6.0(2.6-13.0)$ & $0.41(0.16-1.06)$ & $0.34(0.12-0.96)$ * \\
\hline \multicolumn{10}{|l|}{$\begin{array}{l}\text { Believed that the price } \\
\text { of cigarettes should be } \\
\text { raised to encourage } \\
\text { people to stop smoking }\end{array}$} \\
\hline No & $12.9(10.8-15.3)$ & 1 & 1 & $24.0(19.0-29.9)$ & 1 & 1 & $8.1(6.1-10.7)$ & 1 & 1 \\
\hline Yes & $23.4(18.1-29.6)$ & $2.06(1.55-2.73) * *$ & $2.04(1.57-2.64) * *$ & $36.3(30.9-42.1)$ & $1.80(1.41-2.30) * *$ & $1.17(0.79-1.73)$ & $16.0(11.7-21.6)$ & $2.16(1.67-2.80)^{* *}$ & $2.24(1.36-3.68) * *$ \\
\hline
\end{tabular}

${ }^{\mathrm{a}}$ including PhouThai, Khermou, Khamu, Khmu, Leu, Mong, etc. ${ }^{\mathrm{b}}$ including Christian, Pee, Phi, Phy, Pi, etc. ${ }^{\mathrm{c}}$ International poverty line (http://povertydata.worldbank.org/poverty/

country/LAO) d Adjusted for sex, age groups, education level, income per day, and smoking frequency. ${ }^{*} p<0.05,{ }^{* *} p<0.01,{ }^{* * *} p<0.001$. 


\section{Discussion}

Our study found several individual-level factors that were associated with being a former vs. current smoker or intention to quit, including the beliefs that smoking is harmful or causes illnesses, visiting a healthcare provider in the past year, no exposure to secondhand smoking at workplaces, having a smoking ban in place at home, and noticing media-based messages informing the dangers of smoking or encouraging quitting. Of current smokers, $65 \%$ noticed media-based messages informing the dangers of smoking or encouraging quitting (almost all of these media-based messages were implemented by NTCC), 71\% noticed health warnings on cigarette packages in the past 30 days, and between 63-73\% believed that smoking is seriously harmful to their health or causes illnesses. These proportions are comparable to those in neighboring countries; for example, $67 \%$ of current smokers in Vietnam and Thailand reported thinking about quitting because of health warning labels on cigarette packages [11]. In this study, these factors were associated with approximately three times greater odds of making a quit attempt in the previous year. These results suggest that the relevant tobacco control strategies in Lao PDR (e.g., media campaigns and required health warnings on cigarette packages) have contributed to raising smokers' awareness of the harms of tobacco smoking and may have motivated them to quit. Similar effects of these tobacco control strategies on cessation attempts or intention have also been observed in other member countries that implement the MPOWER measures [11-13]. Specifically, an analysis of GATS data from several developing countries, including Indonesia, Malaysia, Thailand, and Vietnam, showed that large pictorial warnings were associated with a lower cigarette smoking prevalence, particularly among those who had a lower level of education [13]. It is estimated that, among the MPOWER measures, health warnings contribute to preventing the largest number of smoking-attributable deaths (13.3 million, compared to $<1$ million for each of the other MPOWER policies) [14]. Therefore, the Lao NTCC should continue these efforts.

Visiting a healthcare provider in the past year was associated with making quit attempts and quitting smoking. Those Lao smokers who sought healthcare might have had a tobacco-related disease, might have been advised to quit, or might have been more aware of the benefits of quitting. Another plausible explanation was that seeking healthcare and quitting smoking had no causal relationship and might be independent effects of a common cause such as individuals' care for their health. Regardless of the plausibility, this result suggests that a visit to a healthcare provider offers a teachable moment for smoking cessation treatment in Lao PDR, especially when the cause of the visit is related to tobacco use. Unfortunately, most of the current smokers in this study (80\%) reported never receiving advice to quit from a healthcare provider. Although simple advice to quit from a healthcare provider can prompt a cessation attempt, a systematic review showed that simple advice to quit often had a small effect [15]. Another systematic review showed that hospital-based smoking cessation behavioral interventions are effective when supportive contacts continue for at least 1 month [16]. These may explain the lack of an association between cessation intention and ever receiving advice to quit from healthcare providers in this study. In the United States, healthcare providers are recommended to ask patients about tobacco use, advise all identified smokers to quit, and assist them to quit or connect them to a smoking cessation program, such as the 5As approach (Ask, Advise, Assess, Assist, and Arrange) [17] or Ask-Advice-Connect approach [18,19]. These evidence-based approaches have been shown to be effective in increasing cessation attempts and successful cessation. Given the fact that most large healthcare facilities in Lao PDR are public and under the Ministry of Health's supervision, the Lao government may consider establishing a system that motivates healthcare providers to ask patients about their smoking status at each medical visit, advise smokers to quit, and enroll them in an evidence-based smoking cessation program.

Similar to previous studies on this topic and as expected in the MPOWER policy package [6], exposure to secondhand smoking at indoor workplaces was associated with lesser likelihood to quit smoking. Two-third of smokers in Lao PDR reported exposure to secondhand smoking at indoor workplaces; this proportion is higher than that in Thailand (27\%) and in Vietnam (56\%), and is comparable to that in China (63\%) [11]. Exposure to co-workers who smoked might reinforce the peer 
and subjective norms that favor smoking behavior or might trigger craving in those who are trying to quit. Thus, maintaining and expanding national policies regarding smoke-free working environments may facilitate cessation [6]. Similarly, there is evidence in other countries that the implementation of smoke-free homes increased intention to quit and quitting activity [20]. In this study, most current smokers were married and almost all could smoke at home (97\%). Indeed, $87 \%$ of Lao people reported exposure to secondhand smoke at home, making home the most common place of secondhand smoke exposure in Lao PDR [9]. This prevalence was also the highest in Southeast Asian countries, compared to $78 \%$ in Indonesia (the second highest) and $22 \%$ in Brunei (the lowest) [9]. Thus, national campaigns in Lao PDR should discourage smoking indoors at hometo facilitate cessation in smokers and to protect their families from secondhand smoke.

Several studies in Asian countries that implemented the MPOWER policy package have found that a significant proportion of tobacco users want to quit, yet cessation support services and facilities are often lacking [21,22]. Lao PDR is facing a similar situation. In this study, nearly one-fifth of ever smokers have quit (i.e., became former smokers) and nearly one-third of current smokers made quit attempts in the past year. Despite these encouraging cessation numbers, it is important to note that approximately $90 \%$ of smokers who tried to quit, did so cold turkey without medications or counseling. There is a pressing need for effective, affordable, and widely accessible tobacco cessation treatment programs in Lao PDR to reduce smoking and smoking-related morbidity and mortality.

Although $63 \%$ of former smokers and $45 \%$ of current smokers agreed that the price of cigarettes should be raised to encourage cessation, smokers' income was not associated with cessation or intention to quit, cautioning policy making. In our previous analysis, approximately $12 \%$ of smokers' family incomes were spent on cigarettes [3]. Other studies in low- and middle-income countries, such as Cambodia, have also found that low income or poverty did not appear to decrease the use of tobacco $[23,24]$ or increase cessation [25]. Higher price (i.e., tax) of cigarettes has been widely advocated to prevent smoking initiation or reduce smoking in smokers $[26,27]$. Some other evidence, however, suggest that cigarette tax increase has a small effect in reducing cigarette smoking, particularly among moderate or heavy smokers [28,29]. For heavy smokers, demand for cigarettes or nicotine is more inelastic. As aforementioned, effective and affordable tobacco treatment programs are not currently publically available in Lao PDR [4,7], and the cost of over-the-counter nicotine replacement therapy is equal to or greater than cigarettes and is paid fully out-of-pocket. In the absence of low-cost and effective tobacco treatment services, increases in tobacco taxes may result in a greater proportion of smokers' low household incomes being used to purchase cigarettes. Therefore, it is critical to have affordable and effective tobacco treatment programs in Lao PDR prior to further increases in tobacco taxes.

This study has some limitations. Because the survey is cross-sectional, directions of the relationships are uncertain. In addition, the small unweighted numbers of participants in some subgroups resulted in wide confidence intervals and potentially limited the power to detect associations. Responses to several questions about tobacco-related beliefs were binary (i.e., yes/no) instead of on a Likert-scale, and hence may not accurately reflect nuance degrees in participants' perceptions or opinions.

\section{Conclusions}

Tobacco control policies and campaigns in Lao PDR have contributed to raising smokers' awareness of the harms caused by tobacco smoking and may have increased intentions to quit. Nearly one-third of current smokers attempted to quit in the past year and another $11 \%$ planned to quit in the future. There is a pressing need for effective, affordable, and widely accessible tobacco cessation treatment programs in Lao PDR to help these smokers attain their goal of becoming smoke-free. Also, the Lao PDR government should continue tobacco control policies that demonstrated an association with cessation or intention to quit, such as smoke-free environments and required health warnings on 
cigarette packages, in order to facilitate smoking cessation and prevent smoking-related morbidity and mortality.

Author Contributions: P.X. and K.P. oversaw the survey implementation. P.X., D.D., and L.S. developed measures and supervised data collection. L.T.-H.T. and T.C.B. analyzed the data and prepared the manuscript. M.S.B. provided consultation to the analysis and edited the manuscript. All authors have read and agreed to the published version of the manuscript.

Funding: This work was supported by the Southeast Asia Tobacco Control Alliance (SEATCA) and in part by a US National Cancer Institute (NCI) grant (1 R21 CA253600-01) and US NCI Cancer Center Support Grant (P30CA225520) awarded to the University of Oklahoma Stephenson Cancer Center.

Acknowledgments: We would like to thank the Southeast Asia Tobacco Control Alliance (SEATCA) and Lao Ministry of Health for supporting the survey. We appreciate guidance from Mondha Kengganpanich and Sarunya Benjakul at the Faculty of Public Health, Mahidol University, Thailand. We thank Maniphanh Vongphosy and all staff at the Lao-SEATCA office for their kind support. We thank all staff at the Lao National Institute of Public Health for their hard work in data collection. TCB and MSB are supported by a grant from the Oklahoma Tobacco Settlement Endowment Trust (092-016-0002) and in part by the National Cancer Institute Cancer Center Support Grant P30CA225520 awarded to the University of Oklahoma Stephenson Cancer Center. The content is solely the responsibility of the authors and does not necessarily represent the official views of the funding agencies.

Conflicts of Interest: All authors declare no conflict of interest.

\section{References}

1. U.S. National Cancer Institute and World Health Organization. The Economics of Tobacco and Tobacco Control; U.S. Department of Health and Human Services, National Institutes of Health, National Cancer Institute, and World Health Organization: Bethesda, MD, USA, 2016.

2. World Health Organization. Tobacco-Fact Sheet. Available online: http://www.who.int/mediacentre/ factsheets/fs339/en/ (accessed on 15 April 2020).

3. Xangsayarath, P.; Douangvichith, D.; Siengsounthone, L.; Phandouangsy, K.; Tran, L.T.-H.; Bui, T.C. Tobacco use in Lao People's Democratic Republic: Results from the 2015 National Adult Tobacco Survey. Tob. Prev. Cessat. 2019, 5. [CrossRef] [PubMed]

4. World Health Organization. WHO Report on the Global Tobacco Epidemic 2017-Country Profile-Lao People's Democratic Republic; World Health Organization: Geneva, Switzerland, 2017.

5. World Health Organization. MPOWER Brochures and Other Resources. Available online: https://www.who. int/tobacco/mpower/publications/en/ (accessed on 19 October 2019).

6. World Health Organization. MPOWER A Policy Package to Reverse the Tobacco Epidemic; World Health Organization: Geneva, Switzerland, 2008.

7. Ministry of Health. National Adult Tobacco Survey 2015-Lao People's Democratic Republic; Ministry of Health: Vientian, Lao PDR, 2016.

8. Southeast Asia Tobacco Control Alliance (SEATCA). The ASEAN Tobacco Control Report; SEATCA: Jarkata, Indonesia, 2015.

9. Tan, Y.; Dorotheo, U. The Tobacco Control Atlas: ASEAN Region; Southeast Asia Tobacco Control Alliance (SEATCA): Bangkok, Thailand, 2018.

10. World Health Organization. Global Adult Tobacco Survey (GATS). Available online: http://www.who.int/ tobacco/surveillance/survey/gats/en/ (accessed on 19 October 2019).

11. Song, Y.; Zhao, L.; Palipudi, K.M.; Asma, S.; Morton, J.; Talley, B.; Hsia, J.; Ramanandraibe, N.; Caixeta, R.; Fouad, H.; et al. Tracking MPOWER in 14 countries: Results from the Global Adult Tobacco Survey, 2008-2010. Glob. Health Promot. 2016, 23, 24-37. [CrossRef] [PubMed]

12. Palipudi, K.M.; Gupta, P.C.; Sinha, D.N.; Andes, L.J.; Asma, S.; McAfee, T. Social determinants of health and tobacco use in thirteen low and middle income countries: Evidence from Global Adult Tobacco Survey. PLoS ONE 2012, 7, e33466. [CrossRef] [PubMed]

13. Shang, C.; Huang, J.; Cheng, K.W.; He, Y.; Chaloupka, F.J. The association between warning label requirements and cigarette smoking prevalence by education-findings from the Global Adult Tobacco Survey (GATS). Int. J. Environ. Res. Public Health 2017, 14. [CrossRef] [PubMed]

14. Levy, D.T.; Li, Y.; Yuan, Z. Impact of nations meeting the MPOWER targets between 2014 and 2016: An update. Tob. Control 2020, 29, 231-233. [CrossRef] [PubMed] 
15. Stead, L.F.; Buitrago, D.; Preciado, N.; Sanchez, G.; Hartmann-Boyce, J.; Lancaster, T. Physician advice for smoking cessation. Cochrane Database Syst. Rev. 2013. [CrossRef] [PubMed]

16. Rigotti, N.A.; Munafo, M.R.; Stead, L.F. Smoking cessation interventions for hospitalized smokers: A systematic review. Arch. Intern. Med. 2008, 168, 1950-1960. [CrossRef] [PubMed]

17. Fiore, M.C.; Jaen, C.R.; Baker, T.B.; Bailey, W.C.; Benowitz, N.J.; Curry, S.J.; Dorfman, S.F.; Froelicher, E.S.; Goldstein, M.G.; Healton, C.G.; et al. Treating Tobacco Use and Dependence: 2008 Update; U.S. Department of Health and Human Services (USDHHS), Public Health Service (PHS): Rockville, MD, USA, 2008.

18. Vidrine, J.I.; Shete, S.; Cao, Y.; Greisinger, A.; Harmonson, P.; Sharp, B.; Miles, L.; Zbikowski, S.M.; Wetter, D.W. Ask-Advise-Connect: A new approach to smoking treatment delivery in health care settings. JAMA Intern. Med. 2013, 173, 458-464. [CrossRef] [PubMed]

19. Vidrine, J.I.; Shete, S.; Li, Y.; Cao, Y.; Alford, M.H.; Galindo-Talton, M.; Rabius, V.; Sharp, B.; Harmonson, P.; Zbikowski, S.M.; et al. The Ask-Advise-Connect approach for smokers in a safety net healthcare system: A group-randomized trial. Am. J. Prev. Med. 2013, 45, 737-741. [CrossRef] [PubMed]

20. Borland, R.; Yong, H.H.; Cummings, K.M.; Hyland, A.; Anderson, S.; Fong, G.T. Determinants and consequences of smoke-free homes: Findings from the International Tobacco Control (ITC) Four Country Survey. Tob. Control 2006, 15 (Suppl. 3), iii42-iii50. [CrossRef] [PubMed]

21. Singh, P.K. MPOWER and the framework convention on tobacco control implementation in the South-East Asia region. Indian J. Cancer 2012, 49, 373-378. [CrossRef] [PubMed]

22. Singh, P.K. Tobacco control in the WHO South-East Asia Region. Indian J. Cancer 2012, 49, 319-320. [CrossRef] [PubMed]

23. Singh, P.N.; Washburn, D.; Yel, D.; Kheam, T.; Job, J.S. Poverty does not limit tobacco consumption in Cambodia: Quantitative estimate of tobacco use under conditions of no income and adult malnutrition. Asia Pac. J. Public Health 2013, 25, 75s-83s. [CrossRef] [PubMed]

24. Singh, P.N.; Yel, D.; Sin, S.; Khieng, S.; Lopez, J.; Job, J.; Ferry, L.; Knutsen, S. Tobacco use among adults in Cambodia: Evidence for a tobacco epidemic among women. Bull. World Health Organ. 2009, 87, 905-912. [CrossRef] [PubMed]

25. Nargis, N.; Yong, H.H.; Driezen, P.; Mbulo, L.; Zhao, L.; Fong, G.T.; Thompson, M.E.; Borland, R.; Palipudi, K.M.; Giovino, G.A.; et al. Socioeconomic patterns of smoking cessation behavior in low and middle-income countries: Emerging evidence from the Global Adult Tobacco Surveys and International Tobacco Control Surveys. PLoS ONE 2019, 14, e0220223. [CrossRef] [PubMed]

26. Katanoda, K.; Jiang, Y.; Park, S.; Lim, M.K.; Qiao, Y.L.; Inoue, M. Tobacco control challenges in East Asia: Proposals for change in the world's largest epidemic region. Tob. Control 2014, 23, 359-368. [CrossRef] [PubMed]

27. Framework Convention Alliance. World No Tobacco Day 2014: Raising Tobacco Taxes a Win-Win for Governments. Available online: https:/www.fctc.org/resource-hub/world-no-tobacco-day-2014-raisingtobacco-taxes-a-win-win-for-governments/ (accessed on 6 May 2020).

28. Maclean, J.C.; Webber, D.A.; Marti, J. An application of unconditional quantile regression to cigarette taxes. J. Policy Anal. Manag. 2014, 33, 188-210. [CrossRef]

29. Callison, K.; Kaestner, R. Do higher tobacco taxes reduce adult smoking? New evidence of the effect of recent cigarette tax increases on adult smoking. Econ. Inq. 2014, 52, 155-172. [CrossRef]

(C) 2020 by the authors. Licensee MDPI, Basel, Switzerland. This article is an open access article distributed under the terms and conditions of the Creative Commons Attribution (CC BY) license (http://creativecommons.org/licenses/by/4.0/). 